\title{
SUBCATEGORIES OF UNIFORM SPACES $\left({ }^{1}\right)$
}

BY

\author{
MICHAEL D. RICE
}

\begin{abstract}
The problem of embedding a topological space as a closed subspace of a product of members from a given family has received considerable attention in the past twenty years, while the corresponding problem in uniform spaces has been largely ignored. In this paper we initiate the study of the closed uniform subspaces of products of metric spaces. In $\$ 1$ we introduce the functor $m$, which is used in $\S 2$ to characterize the closed subspaces of products of metric spaces and separable metric spaces, and the closed subspaces of powers of the open unit interval $(0,1)$. In $\S 3$ we obtain various descriptions of the functor $d$ which associates to each uniform space a closed subspace of a product of metric spaces and establish the equation $m d=d m$. This leads to a characterzation of the completeness of euX, the uniform space generated by the countable $u$-uniform covers, in terms of the completeness of $u X$ and a countable intersection property on Cauchy filters.
\end{abstract}

1. Preliminaries. Throughout $u X$ will denote a set $X$ with a Hausdorff uniform structure $u$ given in terms of covers of $X$ (see [I]). A uniform space $\alpha X$ is fine if $\alpha$ is the largest uniformity on $X$ which has the same uniform topology. To each uniform space $u X$ one may associate a fine uniform space $\alpha X$ with basis the locally finite covers of the form $\left\{\operatorname{coz} f_{s}: f_{s} \in C(X)\right\}$, where $C(X)$ is the family of real valued functions continuous relative $u X$. A uniform space $u X$ is metric-fine (or $M$-fine) if each uniformly continuous function (map) to a metric uniform space remains a map relative the fine uniformity on the metric space (the uniformity with the basis of open covers). There exists a functor $m$ from uniform spaces into $M$-fine spaces which is right adjoint to the inclusion functor from $M$-fine spaces into uniform spaces; equivalently, the $M$-fine spaces form a coreflective subcategory of uniform spaces. Given $u X, m u$ may be considered the smallest $M$-fine uniformity on $X$ larger than $u$. For example, each fine space is $M$-fine, the separable reflection $e u X$ of an $M$-fine space $u X$ is $M$-fine, and $G_{\delta}$-dense subspaces of compact spaces are $M$-fine. The $M$-fine

Presented to the Society, March 27, 1972 under the title Two theorems in uniform spaces; received by the editors November 7, 1972 and, in revised form, February 1, 1974.

AMS (MOS) subject classifications (1970). Primary 54E15; Secondary 54E35.

(1) This work constitutes a portion of a doctoral dissertation written under Anthony W. Hager of Wesleyan University. The author expresses his appreciation for the advice and understanding of Professor Hager. 
definition and the theory of $M$-fine spaces with a basis of countable uniform covers is due to A. W. Hager (see $[\mathrm{H}]_{1}$ ), while the general $M$-fine theory is due to the author (see $[R]_{1}$ ) and $\mathrm{Z}$. Frolik (see $[\mathrm{Fr}]_{1}$ ).

In $[\mathrm{H}]_{1}$ it is shown that for $u X$ with a basis of countable uniform covers $m u$ has the basis of covers of the form $\left\{\operatorname{coz} f_{n}\right\}$, where $f_{n} \in C(u X)$, the family of maps into the real line with the usual uniformity, and $\operatorname{coz} f_{n}=$ $\left\{x: f_{n}(x) \neq 0\right\}$. From this characterization one obtains $m p u=m c u=m e u$ for each $u X$, where $p u(e u)$ has the basis of finite (countable) $u$-uniform covers and $c u$ is generated by $C(u X)$. More generally, in $[R]_{1}$ and $[\mathrm{Fr}]_{1}$ it is shown that $m u$ has the basis of covers of the form $\left\{\operatorname{coz} f_{n} \cap A_{s}^{n}\right\}$, where $f_{n} \in$ $C(u X)$ and $\left\{A_{s}^{n}\right\} \in u$ for each $n$. Using this result one easily shows that the functors $m$ and $e$ commute: $m e=e m$.

Finally, $\mathfrak{C}$ is a reflective subcategory of uniform spaces if $\mathcal{C}$ is a full replete subcategory (morphisms in $\mathbb{C}$ consist of the maps between the objects of $\mathcal{C}$ and if $X \in \mathbb{C}$ with $Y$ uniformly equivalent to $X$, then $Y \in \mathfrak{S}$ ) with the following property: to each uniform space $X$ there corresponds $X_{\mathfrak{G}} \in \mathfrak{G}$ and and a map $X \rightarrow^{r} X_{\mathfrak{G}}$ such that for each map $X \rightarrow^{f} Y$ with $Y \in \mathfrak{C}$, there exists a unique map $X_{\mathfrak{G}} \rightarrow f^{\prime} Y$ such that $f^{\prime} r=f . \pi$ will denote the functor associated with the reflective subcategory of complete uniform spaces (see [GI]), while the Samuel compactification of $u X$ is $\pi p u X$ and will be denoted by $\beta u X$.

2. Basic results. For completeness and comparison we deal first with the closed subspaces of products of complete metric spaces, namely the complete uniform spaces. In the following $\mathfrak{F}_{0}$ will denote a free ultrafilter of respectively closed sets, zero sets (sets of the form $Z(f)=\{x: f(x)=0\}$ for some $f \in$ $C(X)$ ), or $C(u X)$ zero sets, and $\mathfrak{F}$ will be the filter generated by $\mathfrak{F}_{0}$.

THEOREM 2.1. The following statements are equivalent.

(1) $u X$ is complete.

(2) For each $\mathfrak{F}_{0}$ there exists a map $u X \rightarrow^{f} \rho M$ to a complete metric space $\rho M$ such that $f(\Im)$ has no cluster point.

(3) If $p \in \beta u X-X$, there exists a map $u X \rightarrow f \rho$ to a complete metric space $\rho M$ such that $f^{\beta}(p) \in \beta \rho M-M$, where $f^{\beta}$ is the extension of $f$ over $\beta u X$.

We omit the proof that (1) and (2) are equivalent since the techniques are similar to those found in the proof of 2.2. To show (2) equivalent to (3), assume $p \in \beta u X-X$ and let $\mathfrak{F}_{0}$ be the free closed ultrafilter generated by the closed members of a $p u$-Cauchy filter which represents $p$. From (2) there is a map $u X \rightarrow f M$ such that $f(\mathfrak{F})$ has no cluster point; since $f^{\beta}(\mathfrak{F})=f(\mathfrak{F})$ represents $f^{\beta}(p)$ we have $f^{\beta}(p) \in \beta \rho M-M$. Conversely, given $\mathfrak{F}_{0}, \mathfrak{F}$ is $p u$-Cauchy 
(since $p u$ has a basis of finite uniform covers consisting of $C(u X)$ zero sets). If $\mathcal{F}$ represents $p \in \beta u X-X, f(\mathfrak{F})$ has no cluster point, where $f$ is the map guaranteed by (3).

We comment that the equivalence of (1) and (3) has also been obtained by A. W. Hager (see $[R]_{2}$, footnote).

For the next result let $*$ denote the following property of $u X$ : each closed discrete subspace has Ulam nonmeasurable power (see [GJ]).

THEOREM 2.2. The following statements are equivalent.

(1) $\mathrm{meuX}$ is complete.

(2) For each $\mathfrak{F}_{0}$ there exists a map euX $\rightarrow f \rho$ to a separable metric space $\rho M$ such that $f(\mathcal{Z})$ has no cluster point.

(3) For each $\mathfrak{F}_{0}$ there exists a map $u X \rightarrow f \rho M$ to a metric space $\rho M$ such that $f(\S)$ has no cluster point and * holds.

(4) Each ultrafilter of $C(u X)$ zero sets with the countable intersection property is fixed.

(5) euX is a closed subspace of a product of separable metric spaces.

(6) $u X$ is a closed subspace of a product of metric spaces and * holds.

$(1) \rightarrow(2)$ : Given $\mathfrak{F}_{0}$, we see from (1) that $\mathfrak{F}$ is not meu-Cauchy (otherwise $\mathfrak{F}$ converges to $p$ and $\mathcal{F}_{0}$ is fixed at $p$ ), so there exists a cover $\left\{\operatorname{coz} f_{n}: f_{n} \in C(u X)\right\}$ with $\operatorname{coz} f_{n} \notin \mathcal{F}$ for each $n$ from the remarks in $\S 1$. Let euX $\rightarrow^{f=\left(f_{n}\right)} R^{\aleph_{0}}$ be the standard diagonal map; then one shows that $\left\{f\left(\operatorname{coz} f_{n}\right)\right\}$ is an open cover of $f(X)$. If $p \in f(X)$ belongs to $f\left(\operatorname{coz} f_{m}\right)$, then $p$ is not a cluster point of $f(\mathfrak{F})$ since $Z\left(f_{m}\right) \in \mathfrak{F}_{0}$ and $f\left(\operatorname{coz} f_{m}\right) \cap f\left(Z\left(f_{m}\right)\right)$ $=\varnothing$ (for if $Z\left(f_{m}\right) \notin \mathfrak{F}_{0}$, then $Z\left(f_{m}\right) \cap Z(g)=\varnothing$ for some $Z(g) \in \mathfrak{F}_{0}$; hence $Z(g) \subset \operatorname{coz} f_{m}$ would imply $\left.\operatorname{coz} f_{m} \in \mathcal{F}\right)$.

(3) $\rightarrow(1)$ : Suppose that $\&$ is a nonconvergent meu-Cauchy filter; then the members of $(\xi)$ which are respectively closed sets, zero sets, or $C(u X)$ zero sets may be extended to a free ultrafilter $\mathfrak{F}_{0}$ of that type. Let $u X \rightarrow \rho M$ be the (onto) map guaranteed by (3) such that $f(\mathfrak{F})$ has no cluster point. Then meuX $\rightarrow$ me $M=e \alpha M$ is a map and * insures $e \alpha M$ complete (since a metric space with $*$ holding is realcompact (see $[\mathbf{R}]_{2}$ ) which from $[\mathbf{S}]$ is equivalent to $e \alpha M$ is complete). Since $\mathcal{F}$ is meu-Cauchy, $f(\mathfrak{F})$ is e $\alpha$-Cauchy and hence converges to $p \in M$. Let $U$ be an open set containing $p$ such that $f(F) \cap U$ $=\varnothing$ for some $F \in \mathcal{F}$ (since $f(\mathcal{Z})$ has no cluster point) and choose $g \in$ $C(\rho M)$ with $p \in \operatorname{Int} Z(g)$ and $Z(g) \subset U$; since $f(G) \subset$ Int $Z(g)$ for some $G \in\left(\mathcal{Y}, f^{-1} Z(g) \in \mathcal{F}_{0}\right.$ and $f^{-1} Z(g) \cap F=\varnothing$, contradicting the fact that $\mathcal{F}$ is a filter.

(2) $\rightarrow$ (4): If $\mathbb{F}_{0}$ is a free $C(u X)$ zero set ultrafilter, there is a map $e u X \rightarrow f \rho M$ to a separable metric space $\rho M$ such that $f(\mathfrak{f})$ has no cluster 
point. For each $p \in M$ choose an open set $U_{p}$ such that $U_{p} \cap f\left(F_{p}\right)=\varnothing$ for some $F_{p} \in \mathcal{F}_{0}$ and let $\left\{U_{p(i)}\right\}$ be a countable cover of $M$; then $\bigcap F_{p(i)}$ $=\varnothing$ shows that $\mathfrak{F}_{0}$ does not have the countable intersection property.

(2) $\rightarrow(3)$ : This implication is clear except for showing that $*$ holds. One easily shows that (2) implies meuX complete in a manner similar to the proof of (3) $\rightarrow(1)$. Since meu $\subset e \alpha, e \alpha X$ is complete and hence $X$ is realcompact [S] ; thus each closed discrete subspace of $X$ has Ulam nonmeasurable power [GJ].

(4) $\rightarrow$ (5): Let $\left\{f_{s}\right\}$ be the family of maps from euX into separable metric spaces of power less than or equal the power of $X$ and let $e u X \rightarrow f$ $\Pi \rho_{s} M_{s}$ be the usual diagonal embedding. Suppose that $y=\left(y_{s}\right) \in \overline{f(X)}-f(X)$ and let $\mathcal{S}=\left\{f_{s}^{-1}(\bar{V}): y_{s} \in V, V\right.$ open in $\left.M_{s}\right\}$. (S) generates a free $C(u X)$ zero set ultrafilter $\mathfrak{F}_{0}$ (by the nature of $y$ ). By (4) there exists $\left\{Z\left(f_{n}\right)\right\} \subset \mathfrak{F}_{0}$ such that $\bigcap Z\left(f_{n}\right)=\varnothing$. Let $e u X \rightarrow g R^{X_{0}}$ be the diagonal map derived from the $\left\{f_{n}\right\}$; then $M_{s}=g(X)$ and $g=f_{s}$ for some $s$. The technique used in showing (1) $\rightarrow(2)$ now shows that $f_{s}(\mathfrak{F})$ has no cluster point; hence there is an open set $U$ containing $y_{s}$ such that $f_{s}(F) \cap U=\varnothing$ for some $F \in \mathfrak{F}_{0}$. If $y_{s} \in V$ for $V$ open with $\bar{V} \subset U$, then $f_{s}^{-1}(\bar{V}) \notin \mathfrak{F}_{0}$, which is a contradiction.

(5) $\rightarrow$ (2): Assume that $e u X \rightarrow f \Pi \rho_{s} M_{s}$ is a closed embedding of $e u X$ into a product of separable metric spaces. Let $\mathcal{J}_{0}^{\prime}$ be a free closed ultrafilter on $X$ and let $\mathfrak{F}_{0}=f\left(\mathfrak{F}_{0}^{\prime}\right)$. Assume that $p_{s}(\mathfrak{F})$ has a cluster point $y_{s}$ for each $s$. We claim that $y=\left(y_{s}\right)$ is a cluster point of $\mathfrak{F}_{0}$, which contradicts the fact that $\mathfrak{F}_{0}$ is free; hence there is $s$ such that $p_{s}(\mathfrak{F})$ has no cluster point and $p_{s} f$ is the map desired for (2).

In a manner similar to $(5) \rightarrow(2)$ and $(2) \rightarrow(5)$ one can prove $(6) \rightarrow(3)$ and $(3) \rightarrow(6)$, so the equivalence of (1)-(6) is complete.

We comment that the equivalence of (1) and (4) has been obtained in $[\mathrm{H}]_{1}$ and the implication $(2) \rightarrow(5)$ is suggested by $[\mathrm{Z}]_{1}$. The main idea for 2.2 may be traced to [C]. The equivalence of (2) and (5) may also be obtained using results from $[\mathrm{H}]_{2}$ or $[\mathrm{Re}]$.

THEOREM 2.3. The following statements are equivalent.

(1) $\mathrm{muX}$ is complete.

(2) $u X$ is a closed subspace of a product of metric spaces.

The techniques used in the implications $(6) \rightarrow(3)$ and $(3) \rightarrow(1)$ of 2.2 establish implication (2) $\rightarrow(1)$ of 2.3 . To show (1) $\rightarrow$ (2) we use essentially the technique found in proving (4) $\rightarrow(5)$ of 2.2. That technique shows that it suffices to prove the following: (\#) If $\mathfrak{F}_{0}$ is a free $C(u X)$ zero set ultrafilter, 
there exists a map $u X \rightarrow f \rho M$ to a metric space $\rho M$ such that $f(\mho)$ has no cluster point. If $m u X$ is complete, $\mathfrak{F}$ is not $m u$-Cauchy, so from $\S 1$ there exists $\left\{\operatorname{coz} f_{n} \cap A_{s}^{n}\right\} \in m u$, where $f_{n} \in C(u X)$ and $\left\{A_{s}^{n}\right\} \in u$ for each $n$, such that no member of this cover belongs to $\mathcal{J}$. Choose maps $u X \rightarrow^{g_{n}}$ $\rho_{n} M_{n}$ to metric spaces and open covers $U^{n}$ of $M_{n}$ such that $g_{n}^{-1}\left(U^{n}\right)<$ $\left\{A_{s}^{n}\right\}$ and define the map $u X \rightarrow^{n} \Pi \rho_{n} M_{n} \times R^{X_{0}{ }^{n}}$ by $h(x)=\left(g_{n}(x)\right) \times$ $\left(f_{n}(x)\right)$. Write $R^{x_{0}}=\Pi R_{n}$ and let

$$
U=\left\{U^{n} \times \prod_{k \neq n} M_{k} \times R_{n}-\{0\} \times \prod_{k \neq n} R_{k}: U^{n} \in U^{n}, n \in N\right\}
$$

then one easily sees that $U$ covers $h(X)$. An argument similar to the one used in (1) $\rightarrow(2)$ of 2.2 now shows that $h(\mathcal{J})$ has no cluster point. Hence (\#) is established and the proof of 2.3 is complete.

We comment that " $m u X$ is complete and * holds" may now be added to the equivalences of 2.2. In case $u X$ is precompact we can improve (2) of 2.3 in the following way.

THEOREM 24. The following statements are equivalent for $u X$ precompact.

(1) $m u X$ is complete.

(2) $u X$ is a closed subspace of powers of $(0,1)$.

(2) $\rightarrow$ (1) of 2.4 follows from 2.2 ; to show (1) $\rightarrow$ (2) we once again use the technique from (4) $\rightarrow(5)$ of 2.2. Suppose that $u X \rightarrow^{f} \Pi(0,1)_{s}$ is the standard embedding of $u X$, where $C(u X,(0,1))=\left\{f_{s}\right\}$, and suppose $p \in$ $\overline{f(X)}-f(X)$. Let $\left(\mathcal{Y}=\left\{f_{s}^{-1}(\bar{V}): p_{s} \in V, V\right.\right.$ open in $\left.(0,1)\right\}$ generate a free closed ultrafilter $\mathfrak{F}_{0}$. Since $u X$ is precompact, $\mathfrak{F}$ is $u$-Cauchy and therefore represents a point $z$ in $\pi u X-X$. From (1), $\mathfrak{F}$ is not $m u$-Cauchy, so there exists a cover $\left\{\operatorname{coz} g_{n}\right\}, g_{n} \in C(u X), 0 \leqslant g_{n} \leqslant 1 / 2$, with $\operatorname{coz} g_{n} \notin \mathfrak{F}$ for each $n$. Choose $f_{n} \in C(\pi u X), 0 \leqslant f_{n} \leqslant 1 / 2$, such that $X \cap \operatorname{coz} f_{n}=\operatorname{coz} g_{n}$. Since $\mathcal{F}$ represents $z, z \notin \bigcup \operatorname{coz} f_{n}$. Choose $f_{s}$ such that $\operatorname{coz} f_{s}^{\pi}=\bigcup \operatorname{coz} f_{n}$, where

$$
\pi u X \stackrel{f_{s}^{\pi}}{\longrightarrow}[0,1 / 2]
$$

is the extension of $f_{s}$ over $\pi u X$. Since $f_{s}(\mathfrak{f})$ represents $f_{s}^{\pi}(z)=0$ in $[0,1 / 2], f_{s}(\delta)$ has no cluster point in $(0,1)$; in particular there are open sets $U$ and $V$ with $p_{s} \in V$ and $\bar{V} \subset U$ such that $U \cap f_{s}(F)=\varnothing$ for some $F \in \mathcal{F}$. Then $f_{s}^{-1}(\bar{V}) \cap F=\varnothing$ contradicts the fact that both sets belong to ₹. Hence $f$ is a closed embedding.

At this time I should note my debt to the referee for pointing out that the results found in 2.2(1)-(4), 2.3, and 2.4 may be obtained as special cases in the following more general setting. Let $\mathfrak{M}\}$ be a class of metric spaces satisfying 
natural conditions (which are true for all, or separable, or precompact metric spaces). Then mruX is complete if and only if $r u X$ is a closed subspace of a product of members from $\mathfrak{M}$, where $r u X$ is the reflection of $u X$ into spaces generated by their maps to members of $\mathfrak{M}$. This characterization uses essentially the fact that $m u X$ is the supremum of the fine modifications of uniformly continuous pseudometrics. For more general ideas dealing with the $M$-fine definition the reader is referred to the metrically determined subcategories found in $[R]_{1}$. For the purposes of this paper the author has preferred a more restrictive approach; the reader is invited to develop his own generalization of the setting.

COROLlaRY 2.5. Let $u=p \alpha$ be the Čech uniformity on the topological space $X$. Then $u X$ is a closed subspace of powers of $(0,1)$ precisely when $X$ is realcompact.

2.5 follows from 2.4 since $m p \alpha=e \alpha$ and $e \alpha X$ is complete precisely when $X$ is realcompact [S].

Corollary 2.6. Assume that $u X$ is complete and * holds. Then

(1) cuX and euX are each closed subspaces of products of separable metric spaces, and

(2) puX is a closed subspqce of powers of $(0,1)$.

The hypothesis of 2.6 guarantees $m u X$ complete, so $m e u X$ is complete from 2.2 and 2.3; hence (1) follows from 2.2 while (2) follows from 2.4 (since $m p u=m e u)$.

From (2) of $2.6 p \rho R$ ( $R$ the real line) is a closed subspace of a product of powers of $(0,1)$. Notice that $(0,1)$ is not a closed subspace of a product of powers of $p \rho R$; in fact, any metric space embeddable in a closed manner in such a product must be compact (for let $d M \rightarrow f \Pi p \rho R_{s}$ be a closed embedding, where $d M$ is metric and $R_{s}=R$ for each $s$; then $d M \rightarrow^{p_{s} f} p \rho R_{s}$ is a map for each projection $p_{s}$, so $d M \rightarrow^{p_{s} f} \rho R_{s}$ is a map by a theorem from proximity theory [NW]. Hence $d M \rightarrow f \Pi \rho R_{s}$ is a closed embedding, so $d M$ must be complete.)

Corollary 27. Assume that $u X$ has Lindelöf topology. Then

(1) $u X$ and cuX are closed subspaces of products of separable metric spaces, and

(2) puX is a closed subspace of powers of $(0,1)$.

The Lindelöf assumption guarantees that $e u=u$ and $m p u=m c u=m u$ $=\alpha$ (the $m u=\alpha$ is found in $[\mathrm{H}]_{1}$; if $U$ is an open cover, for each $x$ in $X$ choose $f_{x} \in C(u X)$ such that $\operatorname{coz} f_{x} \subset U$ for some $U$ in $U$ and let $\left\{\operatorname{coz} f_{x(i)}\right\}$ be a countable subcover of $\left\{\operatorname{coz} f_{x}\right\}$; from $\S 1 \quad\left\{\operatorname{coz} f_{x(i)}\right\}$ belongs 
to $m u$, so $U$ belongs to $m u$ ). Since $\alpha X$ is complete, (1) and (2) now follow from 2.2 and 2.4 .

Neither 2.6 nor 2.7 can be strengthened to read "cuX is complete". Example $B$ in [GI] is a complete noncompact separable metric space $\rho M$ with $c \rho=p \rho$. In [RR] it is shown that if $u X$ is complete and * holds, and $u X$ has a basis of point finite (resp., star finite) uniform covers, then euX (resp., $(u X)$ is complete. Without the assumption of a special basis for $u$, no significant result in this direction is known; there is only the weak 2.8 and a reformulation of the problem in 3.7.

Corollary 2.8. Assume that $C(u X)$ is closed under inversion and * holds. (If $f \in C(u X), f \neq 0$, then $1 / f \in C(u X)$.) Then $u X$ is complete if and only if muX is complete; in this case cuX is complete.

From $[\mathrm{H}]_{1}$ there exists an $M$-fine uniformity $v$ on $X$ such that $c u=$ $c v$. Then $m c u=m e u=e m u=m c v=e v$ (since $e v X$ is $M$-fine). If $m u X$ is complete, * guarantees $c m u X$ complete (from $[H]_{1}$ or [RR]), so $c u X$ is complete.

3. The functor $d$. Define $d u X$ to consist of the points in $\pi u X$ which are represented by $m u$-Cauchy filters on $X$. Our aim is to show that the class ( 5 of spaces for which $d u X=u X$ is precisely the closed subspaces of products of metric spaces and that the reflection of $u X$ into $\mathcal{C}$ is given by $d u X$. For convenience we call the closed subspaces of products of metric spaces \{metric\} complete.

THEOREM 3.1. $d u X=\left\{p \in \pi u X\right.$ : Each $G_{\delta}$ set containing $p$ meets $\left.X\right\}$.

Suppose that each $G_{\delta}$ set containing $p$ meets $X$ and let $\mathcal{F}$ be a $u$ Cauchy filter on $X$ representing $p$. Let $\left\{\operatorname{coz} f_{n} \cap A_{s}^{n}\right\}$ be a basic $m u$-uniform cover, where $f_{n} \in C(u X)$ and $\left\{A_{s}^{n}\right\} \in u$ for each $n$. Let $\operatorname{coz} f_{n}=$ $\operatorname{coz} g_{n} \cap X$ where $g_{n} \in C(\pi u X)$. As in the proof of 2.4, $p \notin U \operatorname{coz} g_{n}=$ $\operatorname{coz} g$ if $\operatorname{coz} f_{n} \notin \mathcal{F}$ for each $n$; then $Z(g)$ will be a $G_{\delta}$ set missing $X$, which is a contradiction. Hence $\operatorname{coz} f_{m} \in \mathfrak{F}$ for some $m$, and since $\mathfrak{F}$ is $u$-Cauchy $A_{s}^{m} \in \mathfrak{F}$ for some $s$, so we have $\operatorname{coz} f_{m} \cap A_{s}^{m} \in \mathfrak{F}$. If follows that $\mathcal{F}$ is $m u$-Cauchy and $p \in d u X$.

If $p \in d u X$, let $\mathcal{F}$ be a $m u$-Cauchy filter representing $p$ and suppose $p \in V$ for some $G_{\delta} V$ missing $X$. Then $p \in Z(f) \subset V$ for some $f \in C(\pi u X)$. Since $u X \rightarrow^{f \mid X} R-\{0\}$ is a map, $m u X \rightarrow^{f \mid X} \alpha(R-\{0\})$ is a map, so $f(\mathfrak{\mho})$ is $\alpha$-Cauchy and hence converges to $z \neq 0$. Thus $f(\mathfrak{F})$ cannot represent $f(p)$ $=0$, which is a contradiction.

LEMMA 3.2. $(m d u)_{\backslash X}=m u$. 
From functorial considerations $(m d u)_{\mid X} \subset m u$; if $U=\left\{\operatorname{coz} f_{n} \cap A_{s}^{n}\right\} \in$ $m u$ and $\operatorname{coz} g_{n} \cap X=\operatorname{coz} f_{n}$ for $g_{n} \in C(\pi u X)$, we see from 3.1 that $d u X$ $\subset U_{\operatorname{coz} g_{n}}$. If $V^{n}$ is a uniform cover of $d u X$ such that $V_{\mid X}^{n}=\left\{A_{s}^{n}\right\}$, then $U^{\prime}=\left\{\operatorname{coz} g_{n} \cap V: \quad V \in U^{n}\right\} \in m d u$ and $U_{\mid X}^{\prime}=U$ shows $m u \subset(m d u)_{\mid X}$.

LEMMA 3.3. (5) is a reflective subcategory with the reflection of $u X$ into (S given by $d u X$.

The proof of 3.3 is routine using 3.2.

THEOREM 3.4. $(S$ is exactly the \{metric\}-complete spaces; hence the reflection of $u X$ into this category is given by duX. Furthermore, the functors $m$ and $d$ commute: $m d=d m$.

From 2.3 and the definition $\sqrt{ }$ is precisely the \{metric -complete spaces, so the first part of 3.4 follows since there exists a unique reflection functor into a reflective subcategory. For the second part, $m d u X$ is complete and contains $m u X$ as a dense subspace by 2.3 and 3.2 ; hence $\pi m u X=d m u X=m d u X$ by the uniqueness of completion.

For further work in the direction of the results 3.1-3.4 the reader is referred to [HR].

THEOREM 3.5. Each mu-Cauchy filter contains a u-Cauchy filter with the countable intersection property; each u-Cauchy filter with the countable intersection property is mu-Cauchy.

If $\mathcal{F}$ is $m u$-Cauchy, $(\mathcal{H}=\{Z(f) \in \mathfrak{i}: f \in C(u X)\}$ generates a mu-Cauchy filter $\mathfrak{F}^{\prime}$ (since $u X$ has a basis of covers by zero sets of uniform maps). If $Z\left(f_{i}\right) \in \Theta$ and $\bigcap Z\left(f_{i}\right)=\varnothing$, then $\left\{\operatorname{coz} f_{i}\right\}$ belongs to meu with $\operatorname{coz} f_{i}$ $\notin \mathbb{J}$ for each $i$, which is a contradiction; hence $\mathcal{F}^{\prime}$ has the countable intersection property. If $\mathfrak{F}$ is $u$-Cauchy with the countable intersection property, one easily sees that the point $p$ in $\pi u X$ represented by $\mathcal{F}$ belongs to the $G_{\delta}$ closure of $X$ in $\pi u X$; then the proof of 3.1 shows that $\mathcal{F}$ is $m u$-Cauchy.

COROLlaRY 3.6. $u X$ is complete if and only if $m u X$ is complete and each u-Cauchy filter contains a u-Cauchy filter with the countable intersection property.

COROLlary 3.7. The following statements are equivalent.

(1) $u X$ is complete and * holds, and each eu (resp. cu)-Cauchy filter contains an eu (resp. cu)-Cauchy filter with the countable intersection property.

(2) euX (resp. cuX) is complete.

If $u X$ is complete, $m u X$ is complete; hence $e m u X=m e u X=m c u X$ is complete by 2.8 , so the result follows from 3.6. 
There are two closing remarks. First, the condition in 3.6 dealing with Cauchy filters is equivalent to the following condition: each minimal $u$-Cauchy filter has the countable intersection property (for each $u$-Cauchy filter $\mathfrak{F}$ there is a smallest $u$-Cauchy filter $\mathcal{F}_{\min }$ equivalent to $\mathcal{F}$ in the sense that the filter $\left\{F \cup F^{\prime}: F \in \mathcal{F}, F^{\prime} \in \mathcal{F}^{\prime}\right\}$ is $u$-Cauchy).

Secondly, 2.4 and 3.6 show that the precompact spaces for which each Cauchy filter with the countable intersection property converges are precisely the closed subspaces of powers of $(0,1)$. These spaces were first defined in [NJ] and characterized in [HS] as the closed subspaces of powers of $P_{\mathbf{X}_{1}}=$ $[0,1]^{\aleph_{0}}-\{1\}$ with the coarsest possible precompact uniformity. Hence $P_{\aleph_{1}}$ and $(0,1)$ generate the same epi-reflective subcategory of uniform spaces. Corollary 2.5 is also found in $[\mathrm{HS}]$ and $[\mathrm{Z}]_{2}$. In the latter the \{metric \}-complete idea is discussed under the title of weak completeness as it is in $[\mathrm{M}]$.

\section{REFERENCES}

[C] H. H. Corson, The determination of paracompactness by uniformities, Amer. J. Math. 80 (1958), 185-190. MR 20 \#1292.

[Fr $]_{1}$ Z. Frolik, Interplay of measurable and uniform spaces, Proc. Internat. Topology Conference in Yugoslavia, Budova, 1972.

$\left[\mathrm{Fr}_{2} \longrightarrow\right.$, A note on metric-fine spaces, Proc. Amer. Math. Soc. 46 (1974), 111-119.

[GI] S. Ginsburg and J. R. Isbell, Some operators on uniform spaces, Trans. Amer. Math. Soc. 93 (1959), 145-168. MR 22 \#2977.

[GJ] L. Gillman and M. Jerison, Rings of continuous functions, University Ser. in Higher Math., Van Nostrand, Princeton, N. J., 1960. MR 22 \#6994.

$[\mathrm{H}]_{1}$ A. W. Hager, Some nearly fine uniform spaces, Proc. London Math. Soc. (3) 28 (1974), 517-546.

$[\mathrm{H}]_{2} \longrightarrow$, Perfect maps and epi-reflective hulls, Canad. J. Math. (to appear).

[HR] A. W. Hager and M. D. Rice, The commuting of coreflectors in uniform spaces with completion, Czechoslovak. J. Math. (to appear).

[HS] M. Hušek, The class of k-compact spaces is simple, Math Z. 110 (1969), 123126. MR 39 \#6260.

[I] J. R. Isbell, Uniform spaces, Math. Surveys, no. 12, Amer. Math. Soc., Providence, R. I., 1964. MR 30 \#561.

[M] K. Morita, Topological completeness and M-spaces, Sci. Rep. Tokyo Kyoiku Daigaku Sect. A 10 (1970), 271-288. MR 42 \#6785.

[N] S. A. Naimpally and B. D. Warrack, Proximity spaces, Cambridge Tracts in Math. and Math. Phys., no. 59, Cambridge Univ. Press, London and New York, 1970. MR 43 \#3992.

[NJ] O. Njåstad, On real-valued proximity mappings, Math. Ann. 154 (1964), 413 419. MR 29 \#4030.

$[R]_{1}$ M. D. Rice, Metric-fine uniform spaces, J. London Math. Soc. (to appear).

$[R]_{2} \longrightarrow$, A short proof that metric spaces are realcompact, Proc. Amer.

Math. Soc. 32 (1972), 313-314. 
$[R]_{3}$ M. D. Rice, Complete uniform spaces, Proc. Second Internat. Topology Conference, Pittsburgh, Pa., 1972.

[RR] M. D. Rice and G. D. Reynolds, Covering properties of uniform spaces, Oxford Quart. J. Math. (to appear).

[Re] G. D. Reynolds, Ultrafilters and epi-reflective hulls (unpublished manuscript).

[S] T. Shirota, A class of topological spaces, Osaka Math. J. 4 (1952), 23-40. MR 14, 395.

$[Z]_{1}$ P. Zenor, Certain subsets of products of metacompact spaces and subparacompact spaces are realcompact, Canad. J. Math. 24 (1972), 825-829. MR 46 \#8181.

$[\mathrm{Z}]_{2} \longrightarrow$ Extensions of topological spaces (to appear).

DEPARTMENT OF MATHEMATICS, OHIO UNIVERSITY, ATHENS, OHIO 45701

Current address: Department of Mathematics, George Mason University, Fairfax, Virginia 22030 